\title{
Crystal engineering of adaptive smart materials: from mechanical bending to self-healing \\ C. Malla Reddy
}

Department of Chemical Sciences, Indian Institute of Science Education and Research (IISER) Kolkata, Mohanpur 741 246, India cmreddy@iiserkol.ac.in

High crystallinity, although desired in materials for a wide range of high-performance engineering applications, generally comes with undesirable attributes such as high brittleness and fragility [1]. This makes crystalline materials incompatible with many future technologies, such as flexible devices and soft-robotics. Recent progress in crystal engineering has brought into light many possible opportunities to address these issues, enabling the design of adaptive crystalline materials that respond to external stimuli with exceptional qualities [1-7]. For instance, crystals that bend (elastically or plastically), twist, curl, wind, jump, exfoliate, laminate, and explode, under external stresses, such as mechanical stress, pressure, light, heat, solvent, etc., have been shown. On the other hand, until very recent time, self-healing was observed only in soft and amorphous materials, mostly involving approaches that use chemical reactions, diffusion, solvent, vapour, electricity, etc., with typical healing time scales in minutes to weeks [8]. A new self-healing mechanism that we recently introduced [9] in materials science, enables ultrafast, near $100 \%$ autonomous diffusion-less repair in crystalline materials that uses electrostatic surface potentials generated on the freshly created fracture surfaces, inherent to certain types of polar single crystals. My talk will cover structure-property correlation for crystal engineering of adaptive materials.

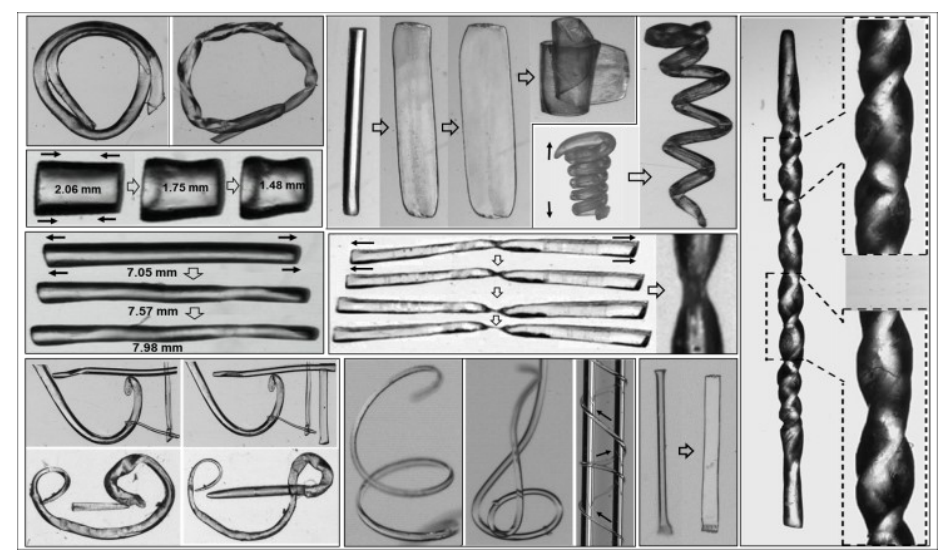

Figure 1. Rotator phase plastic crystals of amino boranes demonstrating extreme ductility and malleability.

[1] Saha, S., Mishra, M. K., Reddy, C. M. \& Desiraju, G. R. (2018) Acc. Chem. Res. 51, 2957.

[2] Reddy, C. M., Gundakaram, R. C., Basavoju, S., Kirchner, M. T., Padmanabhan, K. A. \& Desiraju, G. R. (2005), Chem. Commun. 3945.

[3] Ghosh, S. \& Reddy, C. M. (2012) Angew. Chem. Int. Ed., 51, 10319.

[4] Krishna. G. R., Devarapalli, R., Lal, G. \& Reddy, C. M. (2016) J. Am. Chem. Soc., 138, 13561.

[5] Naumov, P., Chizhik, S., Panda, M. K., Nath, N. K. \& Boldyreva, E. (2015) Chem. Rev., 115, 12440.

[6] Mondal, A., Bhattacharya, B., Das, S., Bhunia, S., Chowdhury, R., Dey, S. \& Reddy, C. M. (2020) Angew. Chem. Int. Ed. 59 ,10971.

[7] Takamizawa, S. \& Miyamoto, Y. (2014) Angew. Chem. Int. Ed. 53, 6970.

[8] Yanagisawa, Y., Nan, Y., Okuro, K. \& Aida, T. (2018) Science, 359, 72.

[9] Bhunia, S., Chandel, S., Karan, S. K., Dey, S., Tiwary, A., Das, S., Kumar, N., Chowdhury, R., Mondal, S., Ghosh, S., Mondal, A., Khatua, B. B., Ghosh, N \& Reddy, C. M. (2021), manuscript in press (accepted).

Keywords: mechanical properties, crystal engineering, plastic crystals, self-healing, piezoelectrics

I thank all my co-authors and collaborators involving in the work presented. DST is acknowledged for Swarnajayanti fellowship (DST/SJF/CSA-02/2014-15) and IISER Kolkata for facilities. 